\title{
Toxicogenomics for transcription factor-governed molecular pathways: moving on to roles beyond classification and prediction
}

\author{
Melvin E. Andersen · Patrick D. McMullen • \\ Sudin Bhattacharya
}

Received: 12 October 2012/Accepted: 13 November 2012/Published online: 25 November 2012

(C) Springer-Verlag Berlin Heidelberg 2012

In this issue of Archives for Toxicology, Jennings et al. (2012) provide a review of transcriptional regulation associated with perturbation of a variety of biological pathways by chemicals. The review focuses on specific transcriptional regulatory networks activated by what the authors refer to as "transcription factor-governed molecular pathways." We will use their terminology throughout our perspective. These pathways include the family of nuclear receptors, several stress-activated transcription factors, and two immunomodulatory transcription factors: STAT and NF- $\kappa$ B. One goal of knowing the specificity of transcriptomic responses from these pathways is development of biomarkers for the "transcription factor-governed pathway" that could support classification or early prediction of likely toxic responses of compounds. In addition to providing a thorough review of toxicogenomics of activation of these pathways, the paper provides two tables showing a sequential process for each pathway from activating events to alterations in transcript levels for target genes. For each pathway, ancillary information includes endogenous ligand, exogenous ligands, inhibitory complexes, DNA target sequences for transcription factor binding, and some target genes. These tables provide a wealth of information related to the context in which the transcription factors function to coordinate information flow from cell exposures to transcription factor-activating ligands/stressors to gene expression. The authors' overview compresses a large amount of information about toxicogenomic responses to activators of transcription factorgoverned pathways in a readily digestible package,

M. E. Andersen $(\varangle) \cdot$ P. D. McMullen · S. Bhattacharya The Hamner Institutes for Health Research, Research Triangle Park, NC 27709-2137, USA

e-mail: MAndersen@thehamner.org highlighting the significant advances in applying microarray technologies in toxicology. This perspective first notes the value of toxicogenomics in organizing information on transcription factor-governed pathways. We then look to a path forward in which toxicogenomics and other technologies will provide detailed knowledge on both the dose-response and structure and function of these transcription factor-governed pathways, thereby supporting a new generation of risk and safety assessment modalities based on cellular assays of the perturbations of these pathways.

\section{Using specific chemical perturbations to evaluate pathway responses}

A simplified description of the transcription factor-governed pathways shows either stress-related or ligand-specific binding as an activating event (Fig. 1). The activated transcription factor $\left(\mathbf{T F}^{*}\right.$ in Fig. 1) then forms an appropriate complex with co-activators and collaborating transcription factors to regulate expression of multiple genes. Multiple transcripts change expression level, some increasing, others decreasing, and provide readout of an integrated cellular response. With rich data sets in hand on toxicogenomic responses to chemicals specifically targeting transcription factor-governed pathways, a library of gene expression studies has become available. In general, the process of identifying pathway targets for an unknown system requires this diverse library of genomic data on compounds that target specific transcription factor-governed pathways. For many pathways, the extent to which a compound is specific to a single target remains poorly understood, complicating some of these studies. The review by Jennings et al. (2012) cata$\operatorname{logs}$ the library of pathway-specific responses that begin to 
allow such analyses. With a new compound, gene expression studies pinpoint one or multiple pathways that appear affected by treatment. In this way, toxicogenomic studies provide information on likely high-dose targets. From other studies in animals or knowledge from human medicine, we can infer toxic responses that may occur in animals upon sufficiently great perturbations of these pathways. These toxicogenomic applications (i.e., predicting likely high-dose responses and classifying chemicals based on these predictions) can speed up hazard identification, reduce animal usage and suffering, and represent a significant advance in the application for this technology. Yet, these contributions remain essentially qualitative. There is great promise in combining toxicogenomics and contributing technologies to provide dose-response information on pathway activation/ toxicology and to gain a much more complete picture of the structure and dose-response characteristics of these pathways. We next look to these opportunities in the context of the progress already apparent from the review of these diverse toxicogenomic studies.

\section{Examining dose-response with genomic changes}

Jennings et al. (2012) point out that gene expression results are amenable to analysis with pathway tools to cluster the altered genes into various Gene Ontology (GO) categories. In addition, many of the papers cited in their review include microarray assessments across dose, for example, studies on phthalate responses in mouse liver (Eveillard et al. 2009). Tools for evaluating dose-response in microarray studies allow examination of the relative responses of different gene groupings across dose. Studies with formaldehyde categorized the dose-response for GO categories in nasal epithelium of rats exposed to formaldehyde (Thomas et al. 2007). This work employed full-genome microarray data and

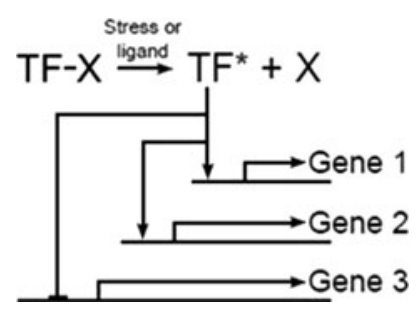

Fig. 1 A simplified depiction of responses in a transcription factorgoverned pathway after perturbation by environmental stress or by an activating ligand. Normally, binding or cellular sequestration keeps the TF in an inactive state. Increased stress or the presence of ligand frees the transcription factor and activates it (designated as $\mathbf{T F} *$ ). $\mathbf{T F}^{*}$ then binds specific DNA-response elements, leading to either enhanced or diminished transcription of $\mathbf{T F}^{*}$-dependent genes. Toxicogenomic tools assess the groups of genes that change due to treatment with chemicals activating specific transcription factorgoverned pathways benchmark dose (BMD) methods (http://www.epa.gov/ raf/publications/benchmarkdose.htm) for assessing doseresponse of individual genes, and GO classification for grouping genes (Beissbarth and Speed 2004; Zhang et al. 2004). This approach provided an aggregate BMD value for significantly enriched genes within various GO categories, including biological processes or molecular functions. There are other dose-response analysis tools available to estimate BMD or an effective concentration causing $50 \%$ responseEC50 (Burgoon et al. 2009; Ji et al. 2009). Many genomic studies have made use of these tools for assessing doseresponse (e.g., Black et al. 2012; Kopec et al. 2010; Thomas et al. 2011). Collectively, these approaches characterize the relative contribution of genes from different ontology categories. It is unlikely that all genes or gene families will have similar dose-response, providing the ability to assess dosedependent transitions in modes-of-action (Slikker et al. $2004 \mathrm{a}, \mathrm{b})$ at the genomic level. The use of toxicogenomic methods to assess BMDs with estimates of statistical bounds for this parameter requires careful consideration of dose spacing in the studies.

\section{Integrated pictures of pathway structure and dynamics}

Toxicogenomics has clearly begun to delineate the downstream consequences of gene expression for specific transcription factor-governed pathways. The field is ready for another defining step forward that will elaborate on the simple scheme in Fig. 1. Using other experimental tools, a much richer view of the pathway structure, pathway dynamics, dose-response, and inter-pathway interactions becomes possible. It is apparent from many studies that a more detailed picture of a transcription factor-governed pathway (Fig. 2) includes multiple layers of control. Transducers relay information from primary transcription factor activation through a broader suite of cooperating TFs. Seven of eight canonical stress pathways (oxidative stress, heat shock response, DNA damage, hypoxia, ER stress, metal stress, and inflammation) have a common structure including sensor, transcription factor, and transducers (Simmons et al. 2009). In these pathways, transduction of information generally occurs through various protein kinases that phosphorylate transcription factors. The group of transcription factors in various combinations control expression of downstream genes. Some of the affected genes have the specific genomic sequences for binding TF* directly (i.e., the consensus binding element on DNA); some bind TF* indirectly through partnering with other transcription factors. With other genes, regulation is completely indirect; the gene does not bind the $\mathbf{T F}^{*}$ protein either directly or indirectly. For estrogen receptor signaling in MCF-7 cells, almost $70 \%$ of genes regulated by estradiol do not contain an 
estrogen response element (Shen et al. 2011). The subsequent control of groups of genes and gene families therefore requires interactions across multiple signal transduction mechanisms, resulting in the activation of several transcription factors.

With many transcription factor-governed pathways, the functional consequences, especially in the upregulation of metabolizing Phase I enzymes or stress-regulating genes, incorporate negative feedback to counteract stress or reduce concentrations of activating ligands. In addition, the initial activation of suites of transcription factors via kinase transducers is usually nonlinear. The transduction steps have nonlinearities (Zhang et al. 2010) arising from multilayer kinases, as with MAPK cascades (Ferrell 1996) or from zero-order ultrasensitivity with specific kinases (Goldbeter and Koshland 1984). Overall, intermediate steps in the control of coordinated gene expression by transcription factor-governed pathways should produce nonlinearities in dose-response through feedback (Zhang and Andersen 2007). Studies to uncover the structure of transcription factor-governed pathways, as depicted in Fig. 2, will also depend on further contributions from toxicogenomics. Pathway studies investigating gene expression following kinase inhibition or employing kinase or transcription factor-knockout studies can pinpoint consequences of alterations in these transduction components in determining the overall structure of the signaling network. However, other technologies, such as phosphoproteomics and genome-wide transcription factor DNAbinding studies, will also be required. The return from expansion of toxicogenomic studies of pathway function into these areas will be a richer appreciation of doseresponse and better use of the knowledge of transcription factor-governed pathways for risk/safety assessment.

\section{Changing directions for toxicity testing and safety assessment}

As noted in the review by Jennings et al., the field of toxicity testing for chemicals is in flux with multiple initiatives in North America and the EU to move away from animal testing to mode-of-action based in vitro assays. Toxicogenomics is an enabling technology in all of these endeavors. The US EPA's ToxCast initiative (Judson et al. 2010) has emphasized prioritization. A suite of

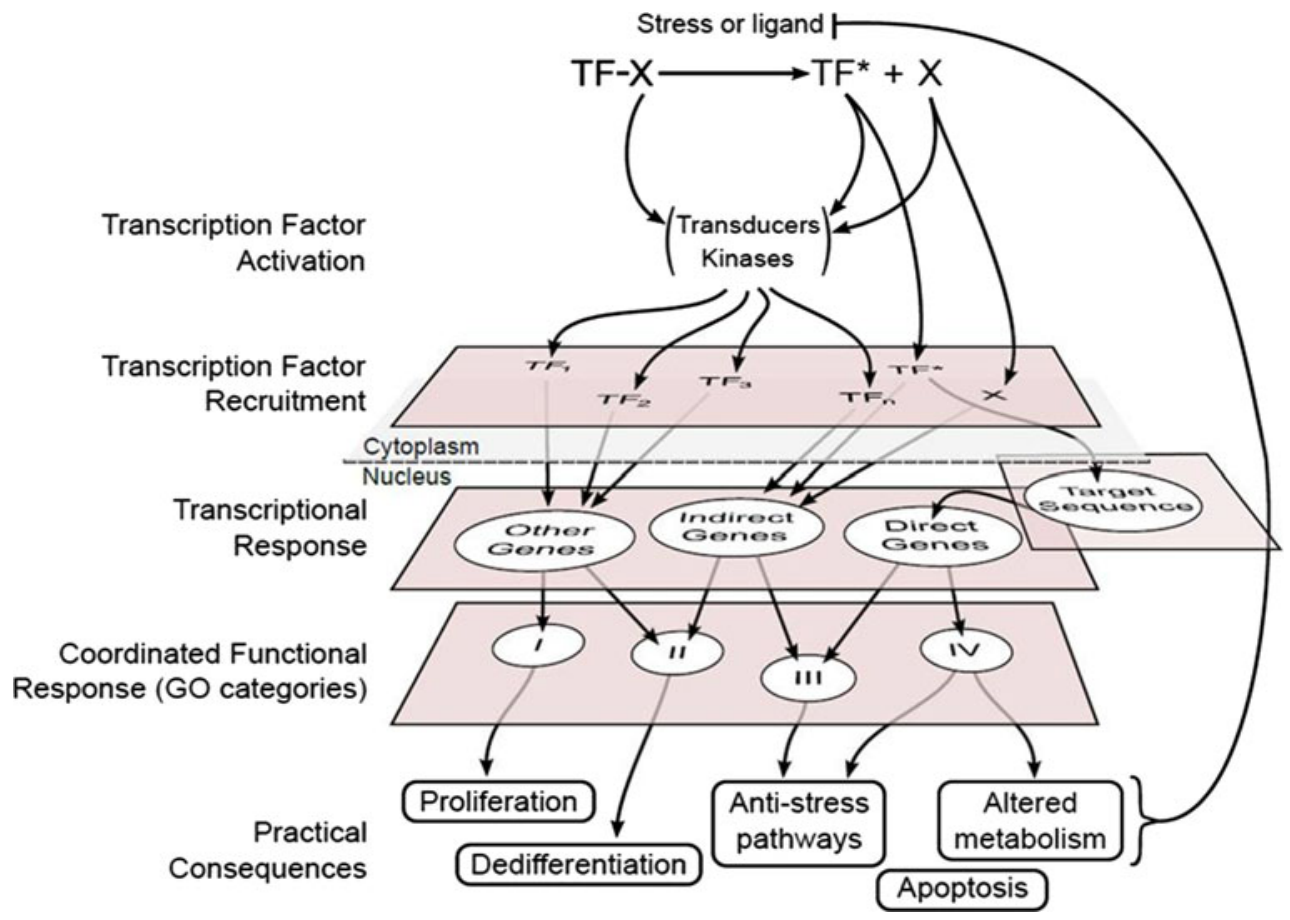

Fig. 2 A more detailed schematic of a transcription factor-governed pathway. These pathways have multiple levels of regulation. Activation of $\mathbf{T F}^{*}$, through binding partners or other transduction partners, activates groups of transcription factors, usually by kinase-mediated phosphorylation. The genes altered by pathway activation include those with $\mathbf{T F}^{*}$ response elements and many others that either bind TF* indirectly or do not interact with $\mathbf{T F}^{*}$ at all. In various combinations, the groups of transcription factors regulate transcription of genes in diverse categories. These genes and gene families regulate cellular consequences and alter phenotype. Frequently, the groups of regulated genes include some intended to reduce the stressor or, especially in the case of the NR activation in liver, to reduce the concentration of active ligand (shown by the negative feedback loop back to the perturbation) 
high-throughput in vitro tests provides a signature of activity that indicates specific targets. The compounds that have the highest association with specific pathways become the highest priority for testing with conventional animal studies. Toxicogenomic results, as described by Jennings et al. (2012), also infer path targets and support similar prioritization. Largely, the European initiatives, such as SEURAT (www.seurat-1.eu) and Predict-IV (www. predict-iv.toxi.uni-wuerzburg.de), also focus on alternatives since legislative pressures in the European Union have restricted use of animals for toxicity testing. The US EPA ToxCast program, at least implicitly, regards animal testing as a preferred methodology for assessing risks; however, the sheer number of compounds requiring testing is so large that some practical means must narrow the number of compounds for testing.

The path forward for using in vitro assays of transcription factor-governed pathways likely requires looking beyond benchmarking in vitro toxicogenomic studies against highdose animal studies. The 2007 National Research Council (NRC) report, Toxicity Testing in the 21st Century: A Strategy and $A$ Vision, argued that in vitro methods, focused on chemical perturbations of "toxicity pathways" in human cells, were the preferred method for toxicity testing and risk assessment. Assays of perturbations of key toxicity pathways would provide results for risk assessment. Transcription factor-governed pathways as described by Jennings et al. are the equivalent of "toxicity pathways" from the NRC report. With the current review of transcription factor-governed pathway components and toxicogenomic outcomes in Jennings et al., we have a glimpse of the organization of 51 such pathways (counting all the individual members of the different nuclear receptor families). A shorter list in the NRC report, compiled to provide examples rather than attempting full coverage of pathways, had 11 examples: 10 of which are in the Jennings et al. list; only one, the osmotic stress pathway with NTFA5 as the central transcription factor, was unique to the NRC list.

Even the larger group of 51 is unlikely to be complete, and other transcription factor-governed pathways are likely to join the list. Notably, absent from the compilation by Jennings et al. were surface receptor-mediated pathways. While it is clear that these receptors themselves do not serve as the transcription factors in the pathways, the consequences of treatment of surface receptors include in most, if not all, cases altered transcriptional regulation (Bhalla et al. 2002). Work with the cannabinoid GPCR receptor showed the regulation of a variety of TFs coordinated by several key kinases-AKT, MAPK1/2, and Src-controls gene transcription (Bromberg et al. 2008; Zorina et al. 2010). Most other pathways that signal through binding to surface receptors also coordinate patterns of gene expression to influence cellular phenotypic consequences. In contemporary toxicology, we have less emphasis on toxicants that preferentially target surface receptors, such as cyclopamine and hedgehog pathway signaling (Heretsch et al. 2010) or belladonna alkaloids acting through acetylcholine neurotransmission at $\mathrm{G}$ protein-coupled receptors. In vitro testing of the toxicogenomic properties and structure of these various pathwaysnuclear receptors, stress and inflammatory responses, and a variety of surface receptor signaling-will continue to elaborate detailed pathway information to empower broader use of in vitro assays of transcription factor-governed assays in risk assessment.

\section{Toxicity pathways}

The 2007 NRC (Krewski et al. 2010; NRC 2007) report discussed using in vitro tests to assess perturbations in "toxicity pathways": normal biological pathways disrupted by treatment with specific chemicals. In reality, there is likely no unique toxicity pathway, only "normal biology" and its perturbation. Toxicity ensues with inappropriate activation or inhibition of normal signaling in cells and tissues with adverse consequences. An overly narrow focus on the term toxicity pathways or "toxicogenomics" may also divert attention from the fact that these studies using chemicals as perturbants probe consequences of inappropriate levels of activity of normal transcription factorgoverned pathways and assess the consequences, including alteration of the transcriptome. Oliver Sacks in his book, The Man who Mistook his Wife for a Hat, talked about neurological conditions as state of loss or excess (Sacks 1985). For us in toxicology, these toxicological conditions might be states of inhibition or activation of these transcription factor-governed pathways. The current review does not emphasize loss of function (e.g., from antagonist ligands or ablation of stress-related transcription factor pathways) as much as pathway activation.

As the toxicogenomics field assesses pathway responses using both losses and excess, the focus of inquiry should be more clearly on pathway biology than on toxicology. Jennings et al. (2012) write in their conclusions that "since the discussed pathways function not only in protection against exogenous compounds, but have evolved to perform physiological roles, their elucidation not only enlightens the toxicological field, but opens the way to a whole new era of pharmaceutical targets, which can potentially revolutionise current concepts in molecular medicine." We join the authors in emphasizing that these pathways function primarily as components of normal biology that are targets for alteration by chemicals. In 1994, Nebert suggested that the drug metabolizing signaling pathways were more likely normal physiological 
pathways whose function was to reduce concentrations of small molecules with biological activity (Nebert 1994). Most of these "drug metabolizing signaling pathways" are nuclear receptor-mediated.

We are seeing a coalescence of activities in toxicogenomics, dose-response modeling of genomic changes, and modeling of transcription factor-governed pathways. The biological pathway, more than the toxic outcome, is becoming the primary focus in understanding the consequences of treatment of cells or tissues with chemicals. It may soon prove that pursuit of dose-response, the dictum from Paracelsus that the dose makes the poison, will push the field of toxicology to make major contributions to understanding biology in our post-genomic world. Here, studies of transcription factor-governed pathways will likely lead to understanding of the risks of chemical exposures and also uncover the dynamics of normal biological signaling networks (as in Fig. 2). Contributions from toxicogenomics, as described by Jennings et al. (2012) with their emphasis on transcription factorgoverned pathways, are enhancing our focus on normal biology and consequences of perturbation of these pathways by different chemicals.

\section{References}

Beissbarth T, Speed TP (2004) GOstat: find statistically overrepresented Gene Ontologies within a group of genes. Bioinformatics 20(9):1464-1465. doi:10.1093/bioinformatics/bth088

Bhalla US, Ram PT, Iyengar R (2002) MAP kinase phosphatase as a locus of flexibility in a mitogen-activated protein kinase signaling network. Science 297(5583):1018-1023. doi:10.1126/science. 1068873

Black MB, Budinsky RA, Dombkowski A et al (2012) Cross-species comparisons of transcriptomic alterations in human and rat primary hepatocytes exposed to 2,3,7,8-tetrachlorodibenzo- $p$-dioxin. Toxicol Sci 127(1):199-215. doi:10.1093/toxsci/kfs069

Bromberg KD, Ma'ayan A, Neves SR, Iyengar R (2008) Design logic of a cannabinoid receptor signaling network that triggers neurite outgrowth. Science 320(5878):903-909. doi:10.1126/science.1152662

Burgoon LD, Ding Q, N'Jai A et al (2009) Automated dose-response analysis of the relative hepatic gene expression potency of TCDF in C57BL/6 mice. Toxicol Sci 112(1):221-228. doi:10.1093/toxsci/ kfp180

Eveillard A, Lasserre F, de Tayrac M et al (2009) Identification of potential mechanisms of toxicity after di-(2-ethylhexyl)-phthalate (DEHP) adult exposure in the liver using a systems biology approach. Toxicol Appl Pharmacol 236(3):282-292. doi:10.1016/j. taap.2009.02.008

Ferrell JE Jr (1996) Tripping the switch fantastic: how a protein kinase cascade can convert graded inputs into switch-like outputs. Trends Biochem Sci 21(12):460-466

Goldbeter A, Koshland DE Jr (1984) Ultrasensitivity in biochemical systems controlled by covalent modification. Interplay between zero-order and multistep effects. J Biol Chem 259(23):1444114447

Heretsch P, Tzagkaroulaki L, Giannis A (2010) Cyclopamine and hedgehog signaling: chemistry, biology, medical perspectives.
Angew Chem Int Ed Engl 49(20):3418-3427. doi:10.1002/anie. 200906967

Jennings P, Limonciel A, Felice L, Leonard MO (2012) An overview of transcriptional regulation in response to toxicological insult. Arch Toxicol. doi:10.1007/s00204-012-0919-y. Accessed 28 Aug 2012

Ji RR, de Silva H, Jin Y et al (2009) Transcriptional profiling of the dose response: a more powerful approach for characterizing drug activities. PLoS Comput Biol 5(9):e1000512. doi:10.1371/journal. pcbi. 1000512

Judson RS, Houck KA, Kavlock RJ et al (2010) In vitro screening of environmental chemicals for targeted testing prioritization: the ToxCast project. Environ Health Perspect 118(4):485-492. doi: 10.1289/ehp.0901392

Kopec AK, Burgoon LD, Ibrahim-Aibo D et al (2010) Automated dose-response analysis and comparative toxicogenomic evaluation of the hepatic effects elicited by TCDD, TCDF, and PCB126 in C57BL/6 mice. Toxicol Sci 118(1):286-297. doi: 10.1093/toxsci/kfq236

Krewski D, Acosta D Jr, Andersen M et al (2010) Toxicity testing in the 21st century: a vision and a strategy. J Toxicol Environ Health B Crit Rev 13(2-4):51-138. doi:10.1080/10937404.2010.483176

Nebert DW (1994) Drug-metabolizing enzymes in ligand-modulated transcription. Biochem Pharmacol 47(1):25-37

NRC (2007) Toxicity testing in the 21st century: a vision and a strategy. The National Academies Press, Washington

Sacks O (1985) The man who mistook his wife for a hat. Touchstone, New York

Shen C, Huang Y, Liu Y et al (2011) A modulated empirical Bayes model for identifying topological and temporal estrogen receptor alpha regulatory networks in breast cancer. BMC Syst Biol 5(1):67. doi:10.1186/1752-0509-5-67

Simmons SO, Fan CY, Ramabhadran R (2009) Cellular stress response pathway system as a sentinel ensemble in toxicological screening. Toxicol Sci 111(2):202-225. doi:10.1093/toxsci/kfp140

Slikker W Jr, Andersen ME, Bogdanffy MS et al (2004a) Dose-dependent transitions in mechanisms of toxicity: case studies. Toxicol Appl Pharmacol 201(3):226-294. doi:10.1016/j.taap.2004.06.027

Slikker W Jr, Andersen ME, Bogdanffy MS et al (2004b) Dosedependent transitions in mechanisms of toxicity. Toxicol Appl Pharmacol 201(3):203-225. doi:10.1016/j.taap.2004.06.019

Thomas RS, Allen BC, Nong A et al (2007) A method to integrate benchmark dose estimates with genomic data to assess the functional effects of chemical exposure. Toxicol Sci 98(1):240-248. doi:10.1093/toxsci/kfm092

Thomas RS, Clewell HJ 3rd, Allen BC et al (2011) Application of transcriptional benchmark dose values in quantitative cancer and noncancer risk assessment. Toxicol Sci 120(1):194-205. doi: 10.1093/toxsci/kfq355

Zhang Q, Andersen ME (2007) Dose response relationship in antistress gene regulatory networks. PLoS Comput Biol 3(3):345-363. doi:e2410.1371/journal.pcbi.0030024

Zhang B, Schmoyer D, Kirov S, Snoddy J (2004) GOTree Machine (GOTM): a web-based platform for interpreting sets of interesting genes using Gene Ontology hierarchies. BMC Bioinform 5:16. doi:10.1186/1471-2105-5-16

Zhang Q, Bhattacharya S, Andersen ME, Conolly RB (2010) Computational systems biology and dose-response modeling in relation to new directions in toxicity testing. J Toxicol Environ Health B Crit Rev 13(2-4):253-276. doi:10.1080/10937404. 2010.483943

Zorina Y, Iyengar R, Bromberg KD (2010) Cannabinoid 1 receptor and interleukin- 6 receptor together induce integration of protein kinase and transcription factor signaling to trigger neurite outgrowth. J Biol Chem 285(2):1358-1370. doi:10.1074/jbc. M109.049841 